\title{
Digital sandbox modelling of Indian collision to Eurasia
}

\author{
Yasuhiro Yamada†*, Atsushi Tanakał and Toshi Matsuoka† \\ † Department of Civil and Earth Resources Engineering, Kyoto University, JAPAN \\ $\neq$ Sumiko, JAPAN \\ * To whom correspondence should be addressed. E-mail:yama@electra.kumst.kyoto-u.ac.jp
}

\begin{abstract}
Summary
Discrete Element Method (DEM) is a powerful tool as a digital sandbox simulator to numerically reproduce fault related structures and to analyse the deformation quantitatively. Similar to analogue sandbox experiments, DEM approximates the geologic body as an assembly of particles that can properly simulate the brittle behaviour of the upper crust. We examined the collision process of the Indian sub-continent to the Eurasian Plate by using the DEM. The simulations reproduced the deformation geometry similar to published analogue experiments. Velocity and stresses of each particle extracted from the simulation were quite unstable showing a characteristic feature of the brittle behaviour of the upper crust. A comparison of these results with GPS and in-situ stress data of the eastern Asia suggests that the ductile deformation of the lower crust and mantle may have major roles to control the real deformation. The results also suggest that Tapponnier's tectonic model may have a strong boundary effect, particularly to the stress field within the model.
\end{abstract}

\section{Introduction}

In order to analyse deformation processes of geologic structures, analogue physical experiments have been performed for a century. In particular, experiments using granular materials (dry quartz sand, micro glass beads, etc.) can properly scale the brittle behaviour of the upper crust, and have been successfully applied to various sedimentary basins, especially by the petroleum exploration industry. Recent techniques such as 'sandbox experiments' utilise Newtonian fluids as well, and the combinations of these two materials allow us to examine a great variety of deformation styles. The knowledge obtained from these experiments has been employed to understand structural development processes and also to predict possible future deformations. The experiments have also been contributing to determine the design of data acquisition of geophysical measurements, the parameters of data processing, and geological interpretations.

Since the material of the sandbox experiments is an assembly of grains, the deformation can be simulated with a numerical technique, the Discrete Element Method (DEM). We believe that DEM can replace at least partly the role of the sandbox experiments and can be applied to various scales of tectonic deformations (Yamada and Matsuoka 2004). This paper presents some results of this simulation which has been applied to the Indian collision to the Eurasia.

\section{DEM simulation}

DEM assumes the geologic body to be a particle assembly (Figure 1). The method requires two steps in calculation cycle to simulate the deformation: the first step to evaluate interaction forces for every particle, and the second step to move these particles according to numerical integration following the Newton's equation of motion for the given external forces. The interaction forces can be evaluated from force-displacement law (Cundall

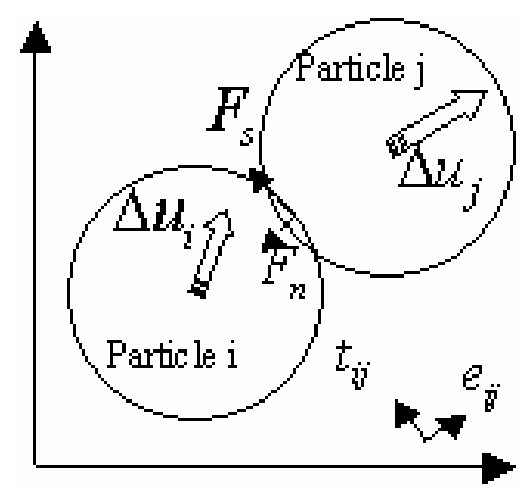

FIGURE. 1 Basic idea of the DEM. The given external forces define the motion of each particle.

and Strack 1979). One of the significant advantages of DEM is that velocity and stresses can be extracted from each particle during deformation. When applied to real geology, these can be compared with GPS and in-situ stress/seismicity data.

\section{Analogue Models}

Lithospheric deformation process of the Indian collision to Eurasia has been investigated by a series of analogue physical experiments (Davy and Cobbold 1988, Tapponnier et al. 1982). Basic assumptions of these experiments included 1) rigid Indian continent, 2) deformable Eurasian Plate, 3) constrained northern and western margins of the experiments, and 4) unconstrained southern and eastern margins.

\section{Simulation Results}

We have conducted a series of DEM simulations following the same assumptions as those of the analogue experiments above to see the deformation styles and the velocity/stress fields of each particle. In all simulations, particle size was randomized to avoid potential weak planes due to the 'perfect' initial packing arrangement by a homogeneous size of the particle. The collision produced progressive development of fault systems, which propagated from the left corner of the indenter to the unconstrained right margin. The outline of the right- hand-side of the particle assembly is broadened to overcome the space problem due to the collision. During the deformation, velocity distribution within the assembly is quite unstable and easily changed the magnitude as well. The region in front of the indenter, however, has no systematic direction, suggesting that the region is highly fragmented. The normal stress distribution suggests that fragmented blocks in the east of the indenter are not highly compressed and this may correspond to the boundary condition of the unconstrained right margin. The normal stress 
in the particles is not homogeneous and some chain-like structures of high stresses are formed from the indenter to the constrained walls. The tangential stress diagram shows that the collision produced a broad sinistral shear band in the particles.

\section{Comparison with Experiments/GPS/In-situ Stress Field}

DEM results produced faulting types similar to the analogue experiments. The faults initiated at the left corner of the indenter and propagated to the right margin of the particle assembly. In the frontal region of the indenter, the layers were thinned in the DEM instead of the thrust faults produced in the experiments. The velocity distributions of the particles are quite unstable during the deformation and commonly showed unsystematic variations, whereas GPS vectors are generally continuous from the collision front. This may suggest that the ductile deformation of the lower crust is a significant control of the Indian collision. The normal stresses of the DEM particles are generally directed to the fixed end-walls and are hardly compared with the real directions of the maximum horizontal compressional stresses.
Conclusions

DEM simulation results are correlated well with the analogue experiments, and also with natural deformations including GPS and in-situ stress data. Therefore, the method can be a useful digital simulator to analyse natural geologic deformations qualitatively

\section{References}

Cundall PA and ODL Strack. 1979. A discrete numerical model for granular assemblies. Geotechnique 29: 47-65

Davy P and PR Cobbold. 1988. Indentation tectonics in nature and experiment. 1. Experiments scaled for gravity. Bull Geol Instit Uppsala NS 14: 129-141

Tapponnier P, G Peltzer, R Armijo, A-Y Le Dain, and P Cobbold. 1982. Propagating extrusion tectonics in Asia: new insights from simple experiments with plasticine. Geology 10:611-616

Yamada Y and T Matsuoka. 2004. Digital Sandbox Modelling using Discrete Element Method (DEM): Applications to Fault Tectonics. In: Sorkhabi $\mathrm{R}$ and Y Tsuji (eds), Faults and Petroleum Traps. Amer Assoc Petrol Geol Mem, in press 